\title{
Canary: An Interactive and Query-Based Approach to Extract Requirements from Online Forums
}

\author{
Georgi M. Kanchev \\ Lancaster University \\ g.kanchev@lancaster.ac.uk
}

\author{
Pradeep K. Murukannaiah \\ Rochester Institute of Technology \\ pkmvse@rit.edu
}

\author{
Amit K. Chopra Lancaster \\ University \\ amit.chopra@lancaster.ac.uk
}

\author{
Pete Sawyer Aston \\ University \\ p.sawyer@aston.ac.uk
}

\begin{abstract}
Interactions among stakeholders and engineers is key to Requirements engineering (RE). Increasingly, such interactions take place online, producing large quantities of qualitative (natural language) and quantitative (e.g., votes) data. Although a rich source of requirements-related information, extracting such information from online forums can be nontrivial.

We propose Canary, a tool-assisted approach, to facilitate systematic extraction of requirements-related information from online forums via high-level queries. Canary (1) adds structure to natural language content on online forums using an annotation schema combining requirements and argumentation ontologies, (2) stores the structured data in a relational database, and (3) compiles high-level queries in Canary syntax to SQL queries that can be run on the relational database.

We demonstrate key steps in Canary workflow, including (1) extracting raw data from online forums, (2) applying annotations to the raw data, and (3) compiling and running interesting Canary queries that leverage the social aspect of the data.
\end{abstract}

\section{INTRODUCTION}

Online interactions between stakeholders and engineers, e.g., on social media and product discussion forums, contain a variety of requirements-related information. Often, such information is unorganized and too noisy to be readily valuable for RE [3]. However, our observations [2] suggest that online discussions have a naturally emerging structure that can be leveraged to extract requirements-related information. A typical discussion starts with a problem description, which can be about a missing or a poorly-implemented feature of a target application. In response to the problem, other users may propose solutions, express support, or rebut the problem or solutions by pointing out unnecessary or unfeasible aspects. In addition to discussions in natural language, online forums include information such as votes and users' reputation.

We develop Canary, a tool-assisted approach for systematically extracting requirements-related information from online forums. Canary addresses two key challenges. It includes (1) an ontology for annotating online forums with entities of interest to RE, including requirements, solutions, and arguments (supports and rebuttals); and (2) a high-level language in which an engineer can query both technical (e.g., requirements) and social (e.g., most supported solution and least controversial requirement) information from online forums.

We demonstrate Canary's key tools, including those for acquiring discussions and annotations, a relational database for storing annotated discussions, and the Canary query compiler.

\section{DESIGN AND IMPLEMENTATION}

In this section, we describe the key steps in Canary and the technologies used in those steps.

1) Data Extraction: The first step in Canary is to extract information from an online forum. For this demo, we focus on Reddit. We used a custom-built java script using the JRAW Java API wrapper version 0.9.0 [1]. Our script extracts all relevant data and metadata from Reddit and stores them in a MySQL database, preserving the comment-reply tree structure.

2) Annotations: The second step is to annotate the raw data acquired from the online form. In our annotation scheme each comment in a discussion can have one of the four annotations of interest: requirement, solution, support, and rebuttal.

In practice annotations can be obtained efficiently and inexpensively via crowdsourcing. For this demo, we employed annotations from two experts (first two authors of this paper). The experts annotated each discussion in three rounds, communicating conflicts after each round, until complete agreement. We store the annotations, too, in a MySQL database.

3) Compiler: Given an annotated discussion, we can write queries in a high-level language to extract requirements-related information. Since the discussions and annotations are in a relational database, we need a compiler to translate queries in the high-level language to SQL.

We implemented a compiler for Canary syntax in Java. We used Eclipse XText (version 2.9) language definition and parsing library. Xtext is a framework that generates a recursive compiler for a given domain language grammar. We programmed the compiler to take queries written in Canary grammar and generate SQL queries. We selected MySQL as a dialect for the compiled queries.

4) Propagation: Forum discussions are naturally nested. Their comment-reply structure creates large trees of interaction. The challenge for Canary is to traverse the whole tree in order to make an assumption about an object of interest. Consider, for example, a requirement followed by two rebuttals; here, the second rebuttal is a rebuttal to a rebuttal and is thus a support to the requirement. Such nesting can be of arbitrary length. Canary's hierarchical queries systematically propagate support and rebuttals, and their corresponding votes.

\section{EXAMPLE QUERIES}

In this section, we describe how users can run queries that follow the Canary grammar. In order to run the code generated 
from the high level queries, we use a MySQL server running on MySQL workbench 6.4 CE.

Next, we show some interesting queries. Figure 1 shows an example discussion using real data from social media on which we run Canary queries. Figure 2 shows the query to select all requirements from the discussion.

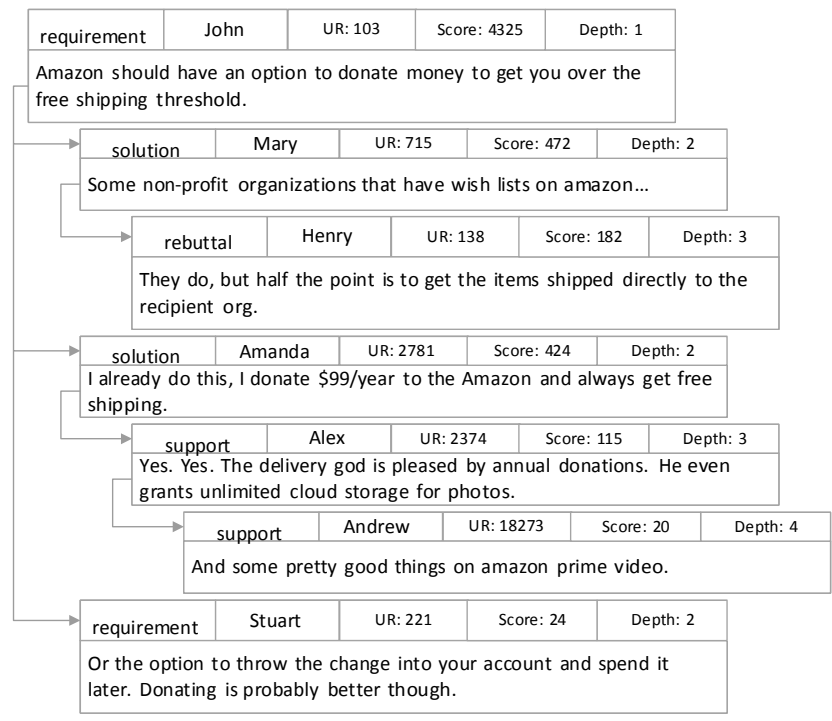

Fig. 1. Example discussion with real data from Reddit.com requirement

\begin{tabular}{|c|c|c|c|c|c|}
\hline text & annotation & user & UR & score & depth \\
\hline donate money & requirement & John & 103 & 4349 & 1 \\
\hline account credit & requirement & Stuart & 221 & 24 & 2 \\
\hline
\end{tabular}

Fig. 2. Canary requirement query

Requirements are the only objects of interest that stand alone. A solution must solve a requirement, and support and rebuttals must be toward another object of interest.

Figure 3 shows a query to select solutions for requirements that mention 'donate money'.

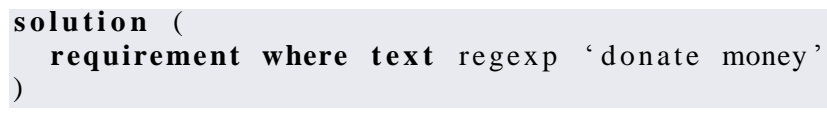

\begin{tabular}{|c|c|c|c|c|c|}
\hline text & annotation & user & UR & score & depth \\
\hline non-profit wish list & solution & Mary & 715 & 290 & 2 \\
\hline Amazon Prime & solution & Amanda & 2781 & 559 & 2 \\
\hline
\end{tabular}

Fig. 3. Canary solution query and results

Figure 3 shows two interesting query elements.

(1) It shows conditions, which can be applied to objects of interest to leverage various metadata associated with them, such as score, natural language text (with support for regular expressions and fuzzy matching), user reputation or role, time of creation, and depth in discussion.

(2) It shows the value propagation brings to queries. In the original discussion '...wish list' has a bigger score than 'Amazon Prime', but with propagated values the score of 'Amazon Prime' increases drastically because of it's two (nested) supporting arguments.

Figure 4, queries for popular requirements; this is an example of what we refer to as aggregator queries. The idea of popular is to select requirements which caused a large amount of positive interaction. As positive interaction we take score, supporting arguments, derived objects. Canary is able to propagate support through nested positive interaction entities (support of support, support of derived, and so on).

popular ( solution ( requirement where regexp
'save address $)$ )
\begin{tabular}{|c|c|c|c|c|c|}
\hline text & annotation & user & UR & score & pop score \\
\hline Amazon Prime & solution & Amanda & 2781 & 559 & $>1$ \\
\hline
\end{tabular}

Fig. 4. Canary aggregator query and results

Popularity is measured using ratios. The calculation is done by summing quantitative data about positive interaction and dividing it by the sum of negative interaction.

$$
\text { Popularity Score }=\frac{\underset{i=0}{n} \mathrm{PV}(\mathrm{i}) \times \mathrm{n}}{\underset{i=0}{m} \mathrm{NV}(\mathrm{i}) \times \mathrm{m}}
$$

where $n$ is the number of children with positive semantics and PV(i) the number of votes for a given child $\boldsymbol{i}$, and $m$ is the number of children with negative semantics and NV(i) the number of votes for a given child $i$.

\section{CONClusion AND Future Work}

Systematic understanding of requirements from the crowd can be crucial for development process of many software projects. In order to achieve that we propose Canary, a toolassisted approach for systematic inclusion of requirementsrelated information from social media. It is designed to combine ontologies from two different fields of research (RE and argumentation) so that queries can fully leverage all aspects of social interaction found in online forums. In its' current implementation Canary is still heavy on manual work, an interesting future direction the application of machine learning algorithms for further automation.

\section{REFERENCES}

[1] JRAW: The Java Reddit API wrapper. https://github.com/thatJavaNerd/ JRAW. Accessed: 2017-03-20.

[2] Georgi Kanchev and Amit Chopra. Social media through the requirements lens: A case study of google maps. First International Workshop on Crowd-Based Requirements Engineering, 2015.

[3] Walid Maalej, Maleknaz Nayebi, Timo Johann, and Guenther Ruhe. Toward data-driven requirements engineering. IEEE Software, 33(1):4854, 2016. 